\title{
Metformin use and young age lung cancer: A case series report
}

\author{
BO DENG ${ }^{1,2^{*}}$, YI WANG ${ }^{1,3^{*}}$, DONG XIE ${ }^{1,4}$, SHAWN M. STODDARD ${ }^{1}$ and PING YANG ${ }^{1}$ \\ ${ }^{1}$ Division of Epidemiology, Department of Health Sciences Research, Mayo Clinic College of Medicine, \\ Rochester, MN 55905, USA; ${ }^{2}$ Department of Thoracic Surgery, Institute of Surgery Research, Daping Hospital, \\ Third Military Medical University, Chongqing 400042; ${ }^{3}$ Division of Preventive Medicine, School of Environmental \\ Science and Public Health, Wenzhou Medical University, Wenzhou, Zhejiang 325035; ${ }^{4}$ Department of \\ Thoracic Surgery, Shanghai Pulmonary Hospital, Tongji University, Shanghai 200433, P.R. China
}

Received January 12, 2015; Accepted September 24, 2015

DOI: $10.3892 / 01.2016 .4307$

\begin{abstract}
Metformin, a widely-prescribed antihyperglycemic drug for the treatment of diabetes mellitus type 2(DM-II), has been demonstrated to be antineoplastic in vivo and in vitro. However, various preclinical and epidemiological studies investigating the effects of metformin on lung cancer have obtained inconclusive results. The aim of the present study was to retrospectively investigate the effects of metformin, for the treatment of diabetes mellitus type 2 (DM-II), on the onset of lung cancer. In the present study, the pathological features of ten consecutive young age lung cancer cases, aged between 15 and 45 years old at the time of diagnosis and exhibiting existing primary DM, were investigated using the Mayo Clinic Lung Cancer Cohort database. Amongst this cohort, there were 2 cases of DM type 1 (DM-I) and 8 cases of DM-II. Of these patients, two exhibiting adenocarcinoma and DM-II had not been administered metformin; however, 1 patient exhibiting lymphoma and 4 patients with pulmonary neuroendocrine tumors (NETs) had been administered metformin at least 12 months prior to lung cancer diagnosis. The remaining 3 patients exhibiting NETs and DM-II had been treated with insulin therapy. The present study hypothesized that the high proportion of NETs observed in the cases of metformin-treated DM-II was unlikely to be a random event. It was suggested that metformin treatment was not effective in the prevention of pulmonary NETs, and that metformin may instead induce the occurrence of NETs via as yet unknown signaling pathways. The present hypothesis may potentially serve as a novel indicator for the requirement to
\end{abstract}

Correspondence to: Professor Ping Yang, Division of Epidemiology, Department of Health Sciences Research, Mayo Clinic College of Medicine, $2001^{\text {st }}$ Street SW, Rochester, MN 55905, USA

E-mail: yang.ping@mayo.edu

${ }^{*}$ Contributed equally

Key words: metformin, lung cancer, neuroendocrine tumors monitor young patients with diabetes, who are being treated with metformin, for the occurrence of pulmonary NETs.

\section{Introduction}

Metformin (1,1-dimethylbiguanide hydrochloride), a widely-prescribed antihyperglycemic drug and first-line therapy for the treatment of diabetes mellitus type 2 (DM-II), has demonstrated notable antineoplastic effects in vivo and in vitro (1). A growing body of evidence has suggested that metformin may potentially reduce the risk of developing a number of types of cancer, including breast (2), colorectal (3), prostate (4) and lung cancer (5). However, Smiechowski et al (6) investigated 8572 patients with DM-II, including 808 lung cancer cases and 7764 controls, and did not identify a significant role of metformin in the risk of lung cancer [odds ratio (OR), 0.94; $95 \%$ confidence interval (CI), 0·76-1·17]. A recent meta-analysis incorporating 11 studies also did not observe a significant association between metformin and lung cancer risk (OR, 0.99; 95\% CI, 0.87-1.12) (7). A common caveat of the aforementioned studies was a lack of consideration of the age and subtypes of lung cancer.

Compared with old patients, young age lung cancer (YALC) cases had a higher proportion of women and were prone to adenocarcinoma development and distant metastases (8). Furthermore, a cohort demonstrated that younger patients had a higher proportion of adenocarcinoma, a higher proportion of stage I disease and a lower proportion of stage III disease (9). The aforementioned results demonstrate the varying clinical characteristics of YALC $(8,9)$.

With regard to young age DM, a previous study reported that the majority of patients were diagnosed during puberty as obese or at risk for obesity (10); this was considered to occur as a result of a variety of genetic conditions (for example maturity-onset diabetes) (11). Such findings support the notion that young age DM presents with specific characteristics that differ from those observed in older cases $(10,11)$.

To the best of our knowledge, there are a limited number of available case reports that focus on the high risk of neuroendocrine tumors (NETs), including carcinoid tumors and small cell lung cancers (SCLC), in YALC and DM treated with oral metformin $(6,12)$. In the present study, patients with YALC 
and DM were investigated. When compared with patients with typical lung cancer (in the sixth to eighth decade of life), it was hypothesized as unlikely that the YALC and DM patients had been differentially impacted by environmental carcinogen exposure (13). If proved to be correct, this may indicate that DM or the treatment of DM with metformin may be associated with increased risk of a specific subtype of lung cancer, (e.g. NETs), which have distinct features of clinical behavior, epidemiology, treatment and prognosis (14).

\section{Case series report}

In the present study, the Mayo Clinic Lung Cancer Cohort database, established in the Epidemiology and Genetics of Lung Cancer research program (15-17), was used to identify 571 consecutive patients with pathologically diagnosed YALC treated at the Mayo Clinic College of Medicine (Rochester, MN, USA) between 1997 and 2011, who were <45 years old at the time of primary lung cancer diagnosis (Table I). Written informed consent was obtained from all patients. Samples were obtained at the time of tumor diagnosis. Formalin-fixed paraffin-embedded samples (if available after diagnosis), slides (for diagnosis) or both were stored at the Department of Laboratory Medicine and Pathology, Mayo Clinic College of Medicine and patients were followed up for 10 years after diagnosis (18). Among the 571 patients selected, 278 (48.7\%) exhibited adenocarcinoma, 76 (13.4\%) carcinoid tumors, $52(9.1 \%)$ squamous carcinoma, 34 (6.0\%) SCLC and 131 (22.9\%) possessed other or unspecified cell type tumors. A review of patient medical history revealed that $10 / 571$ patients had exhibited primary $\mathrm{DM}$ at least one year prior to lung cancer diagnosis (Table I); specifically, there were 2 cases of DM type 1 (DM-I) and eight of DM-II. Three notable observations were made regarding these patients: i) $8 / 10$ patients were overweight or obese, as determined by their body mass index (BMI; BMI, >24.99; Table II); ii) 5/8 patients with DM-II (62.5\%) exhibited pulmonary NETs, including carcinoid tumors and SCLC, which was a higher proportion than that observed in the non-diabetic patients (19.4\%; $5 / 8$ vs. $104 / 561$; Fisher's test, $\mathrm{P}<0.05)$; and iii) most notably, 4 patients exhibiting NETs and one with lymphoma (Table II) had received metformin for the treatment of DM-II. By contrast, the two patients exhibiting adenocarcinoma and DM-II had not been administered metformin. Although 5/8 patients with DM-II treated with metformin were current or former smokers, these patients developed lung cancer 20-30 years earlier in life than the majority of patients, who are lifelong heavy smokers. The proportion of pulmonary NETs in metformin treated patients was significantly higher than those who did not receive metformin treatment (4/5 vs. 106/566; Fisher's test, $\mathrm{P}<0.05$ ).

\section{Discussion}

The incidence of pulmonary NETs has been reported to be $\sim 21 / 100,000$ per year in the Caucasian population of Denmark (14). This figure includes all Caucasian adults; however, the incidence in the young adult population may be lower. To the best of our knowledge, no such published data is currently available. The high proportion $(4 / 5 ; 80 \%)$ of pulmonary NETs observed in patients with metformin-treated DM-II
Table I. Clinicopathological characteristics of 571 consecutively diagnosed young age lung cancer patients.

\begin{tabular}{lr} 
Parameter & Patients, $\mathrm{n}(\%)$ \\
\hline Age at diagnosis, years & \\
Mean (SD) & $39.1(5.1)$ \\
Range & $17.0-44.0$ \\
Gender & \\
Female & $309(54.1)$ \\
Male & $262(45.9)$ \\
Pathological cell type & \\
Adenocarcinoma & $278(48.7)$ \\
Squmaous & $52(9.1)$ \\
Small cell & $34(6.0)$ \\
Carcinoid & $76(13.3)$ \\
Other & $131(22.9)$ \\
Tumor stage & \\
I & $85(14.9)$ \\
II & $35(6.1)$ \\
III & $147(25.7)$ \\
IV & $251(44.0)$ \\
Limited (small cell) & \\
Extensive (small cell) & b \\
Unknown & $16(2.8)$ \\
Tumor grade & $17(3.0)$ \\
Well differentiated & $20(3.5)$ \\
Moderate differentiated & \\
Poor/undifferentiated & $65(11.4)$ \\
Ungradeable & $141(24.7)$ \\
Smoking status & $191(33.4)$ \\
Never-smoker & $174(30.5)$ \\
Former-smoker & \\
Current-smoker & $197(34.4)$ \\
Unknown & $118(20.7)$ \\
& $251(44.0)$ \\
& $5(0.9)$
\end{tabular}

${ }^{\text {aT }}$ Tumors confined to the hemithorax. ${ }^{b}$ Tumors found outside of the hemithorax. 'Grading was not possible due to insufficient tissue samples. Tumors were staged according to the American Joint Committee on Cancer 2010 Cancer Staging Manual (19). SD, standard deviation.

identified in the present study was suggested to be unlikely to be a random event, based on logical suppositions. Regarding the proposed favorable effects of metformin in a number of types of cancer, a suggested potential underlying mechanism is the activation of the liver kinase B1 (LKB1) 5' adenosine monophosphate-activated protein kinase (AMPK) pathway, and inhibition of the mammalian target of rapamycin (mTOR) downstream signaling pathway by ribosomal protein S6 kinase $\beta-1(20,21)$, which possesses a significant role in carcinogenesis and cancer progression (21). In addition, metformin may indirectly inhibit mTOR in lung tissue by decreasing the activation of insulin-like growth factor-1 receptor (IGF-1R)/insulin receptor, rather than through activation of the LKB1/AMPK 
Table II. Clinical characteristics of patients with young age lung cancer exhibiting primary diabetes mellitus.

\begin{tabular}{|c|c|c|c|c|c|c|c|}
\hline $\begin{array}{l}\text { Age, } \\
\text { years }\end{array}$ & Gender & Tumor type & DM Type & Treatment of DM & $\begin{array}{c}\text { Duration of } \\
\text { treatment, months }\end{array}$ & Smoking status & $\begin{array}{l}\mathrm{BMI}, \\
\mathrm{kg} / \mathrm{m}^{2}\end{array}$ \\
\hline 42 & Male & Neuroendocrine $\mathrm{a}^{\mathrm{a}}$ & 2 & Insulin therapy & 6 & Never & 33.92 \\
\hline 41 & Male & Neuroendocrine ${ }^{a}$ & 2 & Metformin & 20 & Former & 33.67 \\
\hline 42 & Male & Neuroendocrine ${ }^{b}$ & 1 & Insulin therapy & Unknown & Current & 19.56 \\
\hline 42 & Male & Neuroendocrine ${ }^{b}$ & 2 & Metformin & 24 & Former & 39.48 \\
\hline 43 & Male & Neuroendocrine ${ }^{b}$ & 2 & Metformin & $>12$ & Current & 25.94 \\
\hline 38 & Male & Neuroendocrine ${ }^{b}$ & 2 & Metformin & $>12$ & Current & 36.66 \\
\hline 44 & Female & Adenocarcinoma & 2 & Glucotrol $^{\mathrm{d}}$ & 36 & Former & 29.27 \\
\hline 44 & Female & Adenocarcinoma & 2 & None recorded & N/A & Current & 28.2 \\
\hline 43 & Male & Pulmonary lymphoma ${ }^{c}$ & 2 & Metformin & $>12$ & Current & 42.62 \\
\hline 24 & Female & Unclassified carcinoma & 1 & None recorded & $\mathrm{N} / \mathrm{A}$ & Never & 19.41 \\
\hline
\end{tabular}

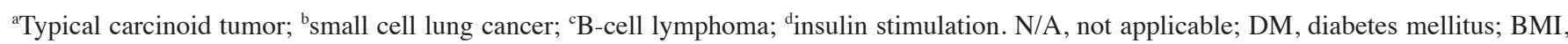
body mass index.

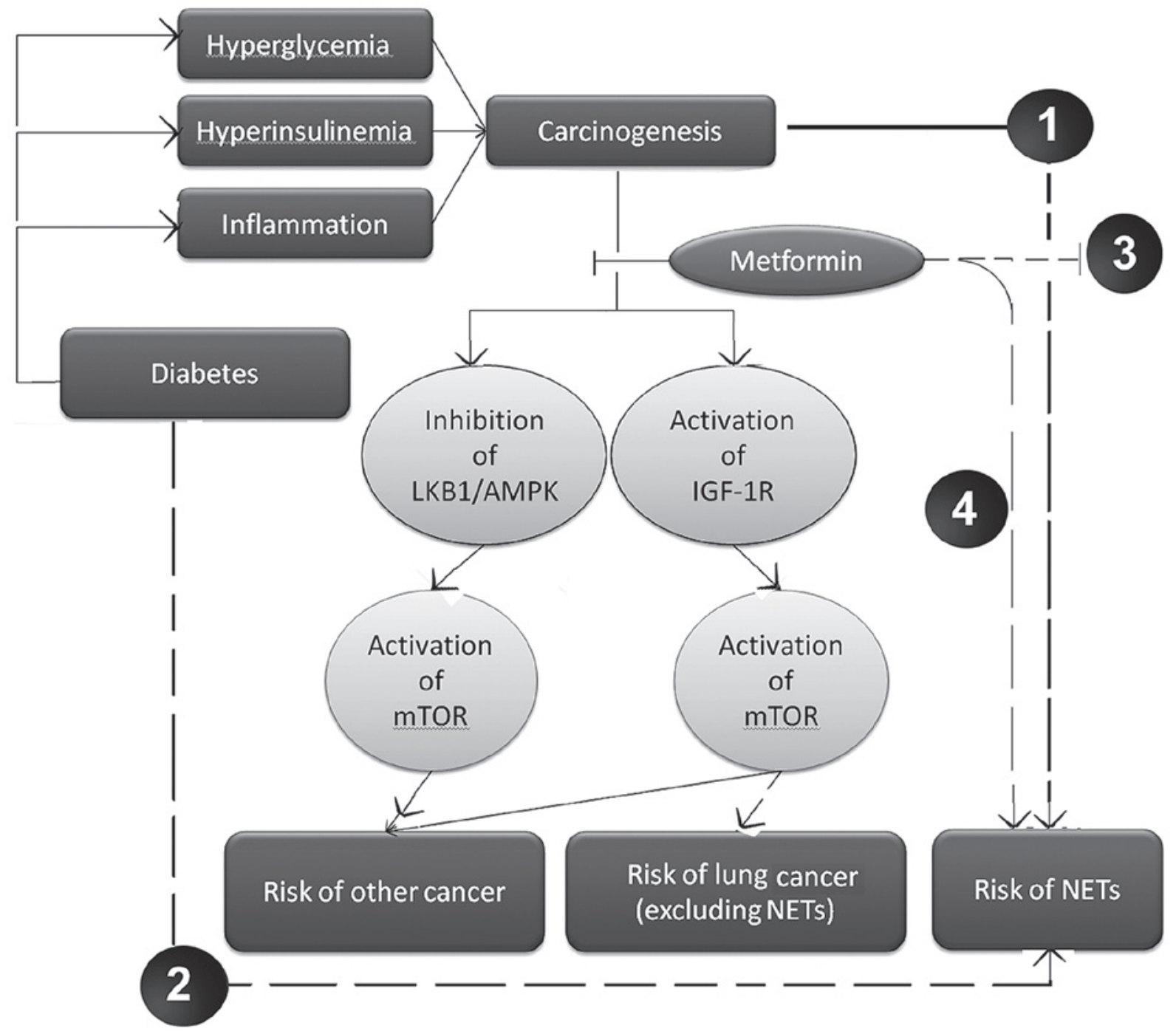

Figure 1. Hypothesis of the potential dual role of metformin in young age lung cancer. (1) Hyperinsulinemia, hyperglycemia and chronic inflammation resulting from diabetes mellitus may be significant carcinogenic mechanisms in NETs. (2) Diabetes mellitus may increase the risk of NETs via as yet unknown pathways, leading to endocrine and metabolic imbalances. (3) In NETs, blocking of IGF-1R by metformin is ineffective as a tumor suppressor and may activate other upstream cascades of mTOR, reducing the antitumor effects. (4) Administration of metformin may induce the occurrence of NETs by as yet unknown signaling pathways. LKB1/AMPK, liver kinase B1/5' adenosine monophosphate-activated protein kinase; IGF-1R, insulin-like growth factor-1 receptor; mTOR, mammalian target of rapamycin; NETs, neuroendocrine tumors. Dashed lines indicate our hypotheses. Solid lines indicate established pathways. 
pathway (22). Furthermore, IGF-1R has been demonstrated not to be expressed in the majority of NET tissues (22), suggesting that the attenuation of IGF-1R activity may be ineffective in the treatment of NETs. Finally, mTOR inhibition has been demonstrated to be capable of inducing upstream receptor tyrosine kinase signaling and activating protein kinase B (Akt) kinase, therefore reducing the antitumor effects of mTOR inhibitors (21). Consequently, the present study hypothesized that, in NETs, the inhibition of mTOR due to the activity of metformin may also activate additional unknown upstream signaling pathways and factors, for example Akt kinase, which subsequently decreases the antitumor effects of metformin.

Although hyperinsulinemia, hyperglycemia and chronic inflammation may be significant mechanisms in the neoplastic process of a number of types of cancer, the carcinogenic mechanisms induced by DM in NETs remain to be elucidated (23). A Taiwanese population-based study revealed that diabetes was associated with a significantly higher risk of lung cancer (24). In addition, a correlation between DM and pancreatic NETs has been reported (25). Therefore, we hypothesize that DM may present a major risk factor for the development of pulmonary NETs, however, further investigation is required. To the best of our knowledge, summarizing all evidence available to date, and as depicted in Fig. 1, the present study hypothesized that metformin was able to decrease IGF-1R expression in lung tissues, consequently inhibiting the downstream mTOR signaling pathway and decreasing the risk of lung cancer (excluding the subgroup of NETs). However, the blockage of IGF-1R activity by metformin is ineffective in NETs. Furthermore, the current study hypothesized that mTOR inhibition, following administration of metformin, may induce the activation of additional upstream oncogenic cascades.

The occurrence of NETs involves unknown signaling pathways (Fig. 1). Previous studies have demonstrated that the use of metformin is not associated with overall lung cancer risk $(6,12)$. However, after stratifying the results by lung cancer subtype, it was found that a longer duration of metformin treatment may be associated with higher risk of small cell carcinoma (12). However, in this study, a high proportion of NETs were observed in the cases of metformin-treated DM-II. Thus, if the results of the present study are able to be verified, they may be useful as a novel alert for the significance of monitoring young patients with diabetes, particularly those who are overweight and receiving metformin, who may possess an increased risk of developing pulmonary NETs.

\section{Acknowledgements}

The present study was supported by the USA National Institute of Health (grant nos. R03 CA77118, R01 CA80127 and R01 CA84354), as well as the Mayo Clinic Foundation (grant no. 300-92021) and Third Military Medical University (MiaoPu project; grant no. MP2012-6-20). The authors would like to thank Ms. Susan Ernst, M.A., for her technical assistance with the manuscript.

\section{References}

1. Wang Y, Dai W, Chu X, Yang B, Zhao M and Sun Y: Metformin inhibits lung cancer cells proliferation through repressing microRNA-222. Biotechnol Lett 35: 2013-2019, 2013.
2. Col NF, Ochs L, Springmann V, Aragaki AK and Chlebowski RT: Metformin and breast cancer risk: A meta-analysis and critical literature review. Breast Cancer Res Treat 135: 639-646, 2012.

3. Currie CJ,Poole CD and Gale EA: The influence of glucose-lowering therapies on cancer risk in type 2 diabetes. Diabetologia 52: 1766-1777, 2009.

4. Wright JL and Stanford JL: Metformin use and prostate cancer in Caucasian men: Results from a population-based case-control study. Cancer Causes Control 20: 1617-1622, 2009.

5. Memmott RM, Mercado JR, Maier CR, Kawabata S, Fox SD and Dennis PA: Metformin prevents tobacco carcinogen-induced lung tumorigenesis. Cancer Prev Res (Phila) 3: 1066-1076, 2010.

6. Smiechowski BB, Azoulay L, Yin H, Pollak MN and Suissa S: The use of metformin and the incidence of lung cancer in patients with type 2 diabetes. Diabetes Care 36: 124-129, 2013.

7. Nie SP, Chen H, Zhuang MQ and Lu M: Anti-diabetic medications do not influence risk of lung cancer in patients with diabetes mellitus: A systematic review and meta-analysis. Asian Pac J Cancer Prev 15: 6863-6869, 2014.

8. Thomas A, Chen Y, Yu T, Jakopovic M and Giaccone G: Trends and characteristics of young non-small cell lung cancer patients in the United States. Front Oncol 5: 113, 2015.

9. Liu M, Cai X, Yu W, Lv C and Fu X: Clinical significance of age at diagnosis among young non-small cell lung cancer patients under 40 years old: A population-based study. Oncotarget: Oct 26, 2015 (Epub ahead of print).

10. Pinhas-Hamiel O and Zeitler P: Clinical presentation and treatment of type 2 diabetes in children. Pediatr Diabetes 8 (Suppl 9): 16-27, 2007.

11. National Institute for Health and Care Excellence: Diabetes (type 1 and type 2) in children and young people: Diagnosis and management. London, UK, 2015. http://www.nice.org.uk/guidance/ ng18/resources/diabetes-type-1-and-type-2-in-children-and-youngpeople-diagnosis-and-management-1837278149317. Accessed February 5,2015.

12. SakodaLC,Ferrara A, Achacoso NS, Peng T, Ehrlich SF, Quesenberry $\mathrm{CP} \mathrm{Jr}$ and Habel LA: Metformin use and lung cancer risk in patients with diabetes. Cancer Prev Res (Phila) 8: 174-179, 2015.

13. Mack JW, Cronin A, Fasciano K, Block SD and Keating NL: Cancer treatment decision-making among young adults with lung and colorectal cancer: A comparison with adults in middle age. Psychooncology: Sep 2, 2015 (Epub ahead of print).

14. Skuladottir H, Hirsch FR, Hansen HH and Olsen JH: Pulmonary neuroendocrine tumors: Incidence and prognosis of histological subtypes. A population-based study in Denmark. Lung Cancer 37: 127-135, 2002.

15. Sun Z, Aubry MC, Deschamps C, et al: Histologic grade is an independent prognostic factor for survival in non-small cell lung cancer: An analysis of 5018 hospital- and 712 population-based cases. J Thorac Cardiovasc Surg 131: 1014-1020, 2006.

16. Jatoi A, Qi Y, Wampfler JA, Busta AJ, Yang P and Mandrekar S: The purported effects of alcohol on appetite and weight in lung cancer patients. Nutr Cancer 63: 1251-1255, 2011.

17. Deng B, Cassivi SD, de Andrade M, et al: Clinical outcomes and changes in lung function after segmentectomy versus lobectomy for lung cancer cases. J Thorac Cardiovasc Surg 148: 1186-1192, 2014.

18. Yang P, Cheville AL, Wampfler JA, Garces YI, Jatoi A, Clark MM, Cassivi SD, Midthun DE, Marks RS, Aubry MC, et al: Quality of life and symptom burden among long-term lung cancer survivors. J Thorac Oncol 7: 64-70, 2012.

19. O'Reilly KE, Rojo F, She QB, Solit D, Mills GB, Smith D, Lane H, Hofmann F, Hicklin DJ, Ludwig DL, et al: mTOR inhibition induces upstream receptor tyrosine kinase signaling and activates Akt. Cancer Res 66: 1500-1508, 2006.

20. Edge S, Byrd DR, Compton CC, Fritz AG, Greene FL and Trotti A (eds): American Joint Committee on Cancer: AJCC Cancer Staging Manual. Springer, New York, 2010.

21. Libutti SK: Therapy: Blockade of IGF-1R - not effective in neuroendocrine tumours. Nat Rev Endocrinol 9: 389-390, 2013.

22. Vlotides G, Tanyeri A, Spampatti M, Zitzmann K, Chourdakis M, Spttl C, Maurer J, Nölting S, Göke B and Auernhammer CJ: Anticancer effects of metformin on neuroendocrine tumor cells in vitro. Hormones 13: 498-508, 2014.

23. Gallagher EJ and LeRoith D: Obesity and diabetes: The increased risk of cancer and cancer-related mortality. Physiol Rev 95: 727-748, 2015.

24. Tseng CH: Diabetes but not insulin increases the risk of lung cancer: A Taiwanese population-based study. PLoS One 9: e101553, 2014.

25. Maxwell JE, O'Dorisio TM, Bellizzi AM and Howe JR: Elevated pancreatic polypeptide levels in pancreatic neuroendocrine tumors and diabetes mellitus: Causation or association? Pancreas 43: 651-656, 2014. 